\title{
Incorporating Intelligence for Overtaking Moving Threatening Obstacles
}

\author{
Mohammed Shuaib ${ }^{1}$ and Zarita Zainuddin ${ }^{2}$ \\ ${ }^{1}$ Department of Computer Sciences and Information, Imam Mohammad Ibn Saud Islamic University, KSA \\ ${ }^{2}$ School of Mathematical Sciences, Universiti Sains Malaysia, Malaysia
}

\begin{abstract}
Crowd management and fire safety studies indicate that the correct prediction of the threat caused by fire is crucial behavior which could lead to survival. Incorporating intelligence into exit choice models for accomplishing evacuation simulations involving such behavior is essential. Escaping from moving source of panic such as fire is of tremendous frightening event while evacuation situation. Predicting the dynamic of fire spreading and the exit clogging are intelligent aspects which help the individuals follow the correct behaviors for their evacuation. This article proposes an intelligent approach to accomplishing typical evacuations. The agents are provided with the ability to find optimal routes that enable them overcome spreading fire. Fire and safe floor fields are proposed to provide the agents with the capability of determining intermediate points to compose optimal routes toward the effective chosen exit. The instinct human behavior of being far from the fire to protect himself from sudden unexpected attack is introduced as essential factor risen in emergency situation. Simulations are conducted in order to examine the simulated evacuees' behavior regarding overtaking the fire and to test the efficiency of making smart and effective decisions during emergency evacuation scenarios.
\end{abstract}

Keywords: Evacuation simulation; fire spread, precaution time; safe floor field.

Received February 25, 2020; accepted June 9, 2020 https://doi.org/10.34028/iajit/17/4A/3

\section{Introduction}

Under the threat of a spreading fire, many factors influence the success of safe evacuations as well as the total evacuation time such as irrational exit choice decisions [5]. Researchers have proved that if the evacuees have not made optimal decisions, such behavior most likely results in disorder and further blockage of the alternative exits [2]. In doing so, it enhances the chance of disasters and crowd stampedes $[14,19]$. Conducting real experiments for achieving this requirement is unrealistic and is an unnecessary danger [26]. This has motivated model developers to develop a large number of simulation models which are separated into two categories. The first one, macroscopic models, is more concerned with the macroscopic behaviors of the whole crowd (see e.g., [12]). The second category comprises of the microscopic models, which are mainly concerned with the detailed interactions among the pedestrians and their physical environment. Among the variety of microscopic models are the Social Force Model (SFM) [10] and the Cellular Automata Models $[1,3]$. Several approaches for calibrating the influential components constituting the simulation crowd models have been proposed $[13,19,30]$.

The model developers have been encouraged to incorporate intelligence factors into the evacuation simulation models or propose independent exit choice models to be combined with the existing evacuation models [7, 17, 21]. Several approaches have been adopted for developing exit choice models. Utility maximization in terms of distance and exit congestion is a common strategy underlying the individual rational decision while escaping from a threatening source (see [22] and references therein). Comprehensive exit choice models were also introduced by involving environmental components such as obstacles and threatening sources and its sequences. Floor field approaches, mostly implemented in the cellular automata models, provide intelligence to the simulated evacuees (denoted here by agents) to consider such components and to select the best exits. The static floor field in which exits are selected with respect to distance disutility constitutes the basis of the floor field approaches [3, 16]. Huang and Guo [11] modified a static floor field to incorporate the effect of internal obstacles on the evacuation process. Zheng et al. [28] introduced the fire floor field to consider the influence of fire spreading rate on the evacuation efficiency. Cao et al. [4] introduced the visibility floor field and temperature floor field to simulate and study pedestrian evacuation under fire emergency. Zheng et al. [29] introduced the smoke floor field and studied the pedestrians' movement behavior when the fire and smoke spread dynamically.

The literature of exit choice models has focused on leaving the fire zone as realistic as possible. However, overtaking dynamic obstacles are not incorporated. In real scenarios, one physical exit structure is a common 
structure, and therefore overtaking dynamic obstacles that hinder the evacuee's egress is indispensable. Besides, experiments indicate that the evacuees are significantly more likely to exit through the familiar door than through a second available exit [15], especially when the situation is under poor visibility or because of the dominance of herding behavior [20]. These cases show the necessity of involving overtaking obstacles hindering motion.

Overtaking behavior is an essential human factor which has been incorporated in normal situations and accordingly produced further realistic crowd simulations. Overtaking immobile obstacles has been treated by researchers for studying the effect of collision avoidance behavior on the evacuation dynamics [11]. Shuaib [25] introduced the collision avoidance field to enable the agents overtake moving obstacles in nonemergency situations. The agents are provided with the capability of selecting intermediate destinations that enable them reaching their preferred destinations based on information given by the collision avoidance field. The agents select the optimal cells to achieve less potential of collision and minimize the distance to the original destination. The proposed model in [25] is integrated in the exit choice model proposed in [22] and simulations are performed to examine the impact of the extended model on introducing further realistic and efficient evacuation.

In this article, to enhance the overtaking capability in emergency situations, we improve the overtaking model proposed in [25] by proposing fire and Safe Floor Fields (SFFs) in order to involve emergency situations with moving threatening sources such as fire. As for the structure of the paper, the next section presents a brief background of the model chosen to be a base for our contribution. We highlight the need for improving the original model to cover further aspects of evacuation. In the third section, we extend the original model to introduce effective overtaking of dynamic threatening obstacles. Finally, the relevant simulations to demonstrate the results of our work are performed.

\section{Crowd Dynamics and Exit Choice Models}

Compared to the macroscopic crowd dynamics models, microscopic models are more superior due to their capability to include detailed interactions between simulated individuals and hence the capability to produce collision avoidance and behaviors beyond, which are the main factors in this article. Among these models, we choose the SFM because of its superiority in producing the above characteristics, as mentioned in the following subsection. On the other hand, the rulebased exit choice model in [22] allows individuals to predict the spreading fire dynamics and includes its effect on the assessment of individuals. This ability is necessary to overcome moving threat obstacles.
Therefore, the combination of the two models is adopted to form the base for our proposed model.

\subsection{Social Force Model and Decision-Making Capability}

The SFM is a spatial-continuous microscopic crowd dynamics model that is characterized with parameters capable to be formulated to accommodate enormous aspects of crowd dynamics: to introduce selforganization phenomena $[9,18]$, to reproduce real-life data [23] and to control various walking behaviors such as penetrating jammed crowd [24] and walking competition $[6,23]$. The latter is an essential factor for understanding faster and slower phenomenon [8, 27]. The simulated individuals in the SFM are self-driven particles. The motion of an individual is mainly caused by social and physical forces exerted by surrounding individuals and objects in the physical environment; the sum of these forces is implemented in a semi-Newtonian equation resulting in the acceleration of the individual's motion:

$$
m_{i} \frac{d \vec{v}_{i}(t)}{d t}=\vec{f}_{i}^{\text {pref }}(t)+\sum_{j} \vec{f}_{i j}^{\text {social }}(t)+\sum_{j} \vec{f}_{i j}^{\text {physical }}(t)+\varepsilon_{i}(t)
$$

Where $\frac{d \vec{v}_{i}(t)}{d t}$ is the acceleration of individual $i$ at time $t ; \varepsilon_{i}(t)$ is the fluctuation term; and the other forces are briefly described as follows:

- The preferred force $\vec{f}_{i}^{\text {pref }}(t)$ which is modeled to express the motivation inside individual $i$ to adapt his actual velocity $\vec{v}_{i}(t)$ to reach his preferred velocity $\vec{v}_{i}^{0}$ at which he prefers to walk:

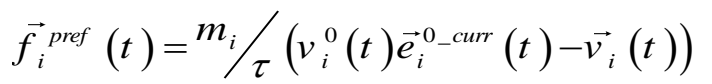

Where $\vec{e}_{i}^{0 \_ \text {curr }}(t)$ is the preferred direction of individual $i$ at time $t, v_{i}^{0}$ is his preferred speed, and $m_{i}$ and $\tau$ represent the mass of the individual $i$ and the relaxation time, respectively.

- The social forces $\vec{f}_{i j}^{\text {social }}(t)$ which are of two types: the repulsion force which represents the model of the repulsive motivation inside agent $i$ to avoid individual $j$ and the attraction force which represents the model of the attractive motivation inside individual $i$ toward individual $j$ [10].

- The physical forces $\vec{f}_{i j}^{\text {physical }}(t)$ denote the pushing and friction forces that arise between two individual in the event of contact that occurs between them.

It is worth noting that Equation (1) produces operational navigation process characterized by instantaneous decisions; SFM has no role in identifying destinations (such as exits). Exit choice behavior is a matter of decision-making aspects that belong to strategic or 
tactical level behavior represented as preferred speed and direction towards the preferred exit, which are referred to here by the preferred velocity $\vec{v}_{i}^{0}$.

\subsection{Rule-Based Exit Choice Model}

The underlying assumption of exit choice behavior is that the evacuees are expected to search for typical emergency exit among alternative exits for safe evacuation. This assumption has been considered along with providing agents with long-ranged awareness of exits located within their sights, ability of investigating the factors influencing the assessment process of choosing the appropriate exit, and decision making capability to direct the motion toward the chosen exit. In [22], the agent accounts for the evacuation time as a disutility in order to decide the exit with minimum disutility. The time disutility $T_{m}^{k}$ of an exit $k$ for agent $m$ is introduced as the maximum disutility of two factors: Troute $_{m}^{k}$ which denotes the time needed to reach the exit $k$ along route $k$, and $T c r o w d_{m}^{k}$ which denotes the time of evacuating a crowd predicted to clogging exit $k$ before arriving the exit. The value of the resulting disutility is affected by the dynamics of the fire; namely, the spreading fire could threaten some exits and consequently affect the choice of exits. Accordingly, the model of time disutility $T_{m}^{k}$ is as follows:

$$
\begin{gathered}
T_{m}^{k}\left(t_{\text {exit }}\right)=\max \left(\text { Troute }_{m}^{k}\left(t_{\text {exit }}\right), \operatorname{Tcrowd}_{m}^{k}\left(t_{\text {exit }}\right)\right) \cdot \operatorname{Risk}_{m}^{k} \\
\operatorname{Troute}_{m}^{k}\left(t_{\text {exit }}\right)=\frac{\operatorname{dis}_{m}^{k}\left(t_{\text {exit }}\right)}{v_{m}^{0}\left(t_{\text {exit }}\right)} \\
\operatorname{Tcrowd}_{m}^{k}\left(t_{\text {exit }}\right)=N_{m}^{k}\left(t_{\text {exit }}\right) T_{\text {eve }}^{k}\left(t_{\text {exit }}\right)
\end{gathered}
$$

Where the factor $\operatorname{Risk}_{m}^{k}$ is to consider the repulsive effect caused by the nearness of fire on the route $k, d i s_{m}^{k}$ is the distance between agent $m$ and exit $k, T_{\text {ave }}^{k}$ is the average rate of passing exit $k$, and $N_{m}^{k}$ is the number of clogging agents predicted by agent $m$. The best exit is the one with minimum disutility:

$$
\text { best_ } k\left(t_{\text {exit }}\right)=\operatorname{index}\left\{\min _{k}\left(T_{i}{ }^{k}\left(t_{\text {exit }}\right)\right)\right\}
$$

The agent decides to replace his current preferred direction $\vec{e}_{m}^{0 \_c u r r}$ in (1) with the direction toward the best exit when the new direction provides the agent with worthwhile utility denoted by the threshold utility, Th_exit as follows:

\section{Decision $_{m}\left(t_{\text {exit }}\right)=$}

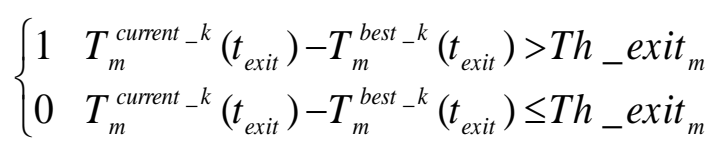

$$
\begin{aligned}
& \vec{e}_{m}^{0 \_c u r r}\left(t_{\text {exit }}\right)=
\end{aligned}
$$

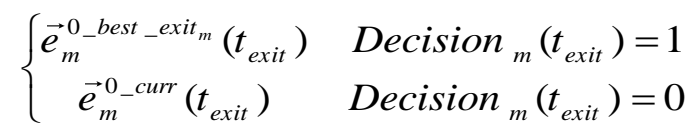

\subsection{Problematic Issue}

However, the rule-based model [22] suffers from a shortcoming; that is when only one exit is available and is obstructed by a fire, the agent avoids the exit because of possible contact with fire rather than trying to overtake the fire as it is the only option. This behavior was exemplified in a simulation with a multi-exit layout (as shown in Figure 1-a), where the fire was initialized in the lower-right section and prepared to spread upward according to simple probabilistic behavior (for the simplicity of simulations) in purpose to hinder the motion of the agents (see Figure 1).

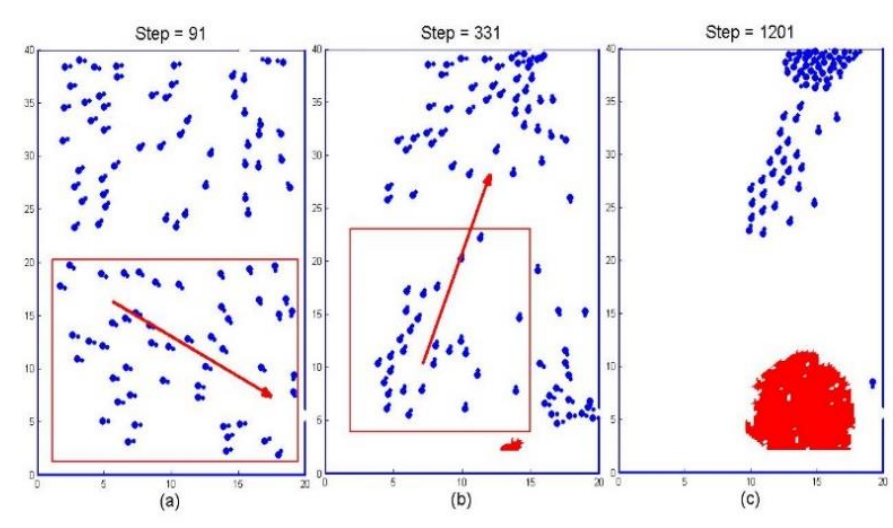

Figure 1. Escaping from the spread of fire based on the exit choice model proposed in [22].

The agents surrounded by the square were initially exiting the lower-right exit to escape the spread of fire. After the prediction of future contact (i.e., the agents' direct routes toward the exit would intersect the fire), the exit is eliminated from agent's subjective choice set of exits. Rationally, however, the threatened individuals would not give up their selected exits if safely overtaking the fire was possible, particularly when the other exits are recognized as having much higher disutility (such as high clogging at the other exits in Figure 1) or are characterized with uncertainty.

Thus, a refinement for the rule-based exit choice model proposed in [22] that can enable the agents to rationally interact with dynamic obstacles and make optimal decisions by overtaking said obstacles is required. We endow the agents in [22] with the capability of overtaking moving obstacles proposed in [25] when the egress through the chosen exit is possible, and its utility is the highest. It is reasonable for this purpose to make use of the floor field to find new routes in order to accomplish the desired overtaking behavior. 


\section{The Extended Model}

\subsection{The Founded Routes Set and Decision for The Optimal Route}

In simple environment such as a large room, it is assumed that each agent has a global view and is familiar with the layouts behind the obstacles. Every agent $m$ has his choice set of visible exits, and correspondingly a choice set of alternative routes (direct routes) connecting the agent directly to the exits. To overtake obstacles hindering the motion toward exit $k$, we endow the agent with the ability of detecting intermediate points. Each of these points connects the agent to the hindered exit via piece-wise segments (subroutes). Reasonably, two intermediate points located on the opposite sides of each obstacle are enough for this purpose (see Figure 2).

Accordingly, we associate each hindered direct route with left and right routes, each of them would be composed of sub-routes: route ${ }_{m}^{k, 0}$ which connects the agent $m$ to the intermediate point, and route $e_{m}^{k, 1}$ which connects the intermediate point to the corresponding exit $k$. Through this work, the agent composes a temporary route set involving left and right founded routes to be associated with the hindered exit $k$ as an alternative of the direct route. The dynamic temporary set are activated when the direct route becomes impeded.

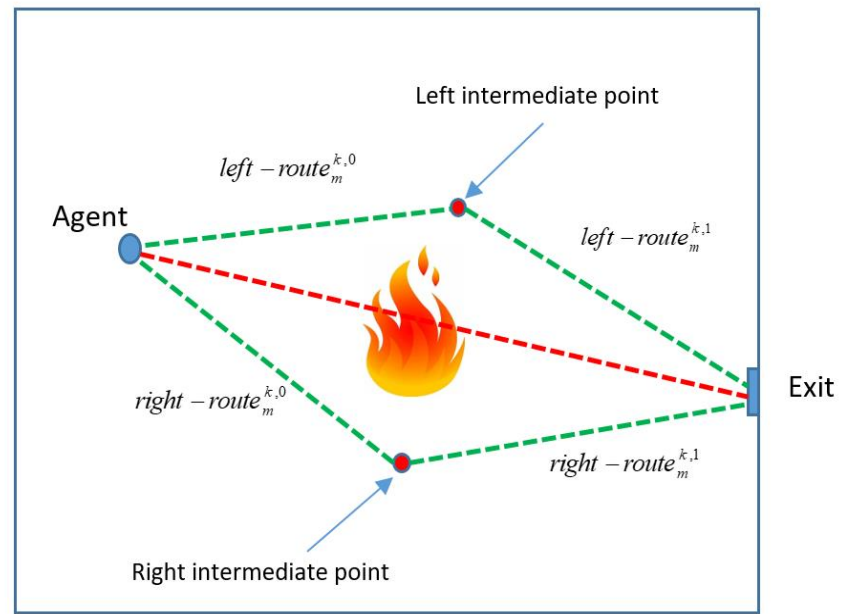

Figure 2. The agent's ability of detecting left and right intermediate points and composing left and right routes.

The agent $m$ would subjugate the temporary set to the disutility test to determine the route with minimum disutility as follows:

$$
k_{-} \text {BestRoute }\left(t_{\text {route }}\right)=\operatorname{index}\left\{\min _{D \in\{\text { lef }, \text { ight }\}} \text { Troute }_{m}^{k-D}\left(t_{\text {route }}\right)\right\}
$$

Where the disutility of each route Troute $_{m}^{k-D}\left(t_{\text {route }}\right)$ is calculated as follows:

$$
\begin{aligned}
& \text { Troute }_{m}^{k-D}\left(t_{\text {route }}\right)=\text { Troute }_{m}^{k-D, 0}\left(t_{\text {route }}\right)+\text { Troute }_{m}^{k-D, 1}\left(t_{\text {route }}\right) \\
& \text { Troute }_{m}^{k-D, l}\left(t_{\text {route }}\right)=\frac{\| \text { route }_{m}^{k-D . l}\left(t_{\text {route }}\right) \|}{v_{m}^{0}\left(t_{\text {route }}\right)}, \quad l \in\{0,1\}
\end{aligned}
$$

In analogy with Equations (7) and (8), at each time step $\Delta t_{\text {route }}$ the agent makes a decision to replace his current route to exit $k$ with the new route (of index $k_{-}$BestRoute). Finally, at each time step $\Delta t_{\text {exit }}$, the route of index $k_{-}$BestRoute would be the representative route for exit $\mathrm{k}$ when performing exitdisutility test subject to Equations (3), (4), (5), (6), (7), and (8).

\subsection{Assigning Intermediate Points}

For detecting intermediate points, it is assumed first that the agent is aware of being far from the fire to protect himself from sudden unexpected attack from fire, temperature or gas. Therefore, each agent has a precaution time $T^{\text {prec }}$. We introduce the fire floor field which is defined as a grid composed of cells of size $0.1 \mathrm{~m} \times 0.1 \mathrm{~m}$. The value of each cell represents the expected time that the fire could reach each cell $(i, j)$. Correspondingly, we introduce the SFF.

The SFF for an agent is used to specify cells in the grid to be considered as attractive (safe) intermediate points to which the agent steers his preferred direction. The agent calculates the expected times $T_{m}^{\text {cell }(i, j)}\left(t_{\text {route }}\right)$ and $T_{\text {fire }}^{\text {cell }(i, j)}\left(t_{\text {route }}\right)$ that he and the fire could reach each cell $(i, j)$, respectively. The SFF is proposed as a dynamic field because of the dynamic spread of fire. Therefore, the agents keep updating the detection process of intermediate points in conjunction with the detection process. The formula that assigns safe values for the corresponding cells is as follows:

$$
\begin{aligned}
& \text { Tsafe }_{m}^{\text {cell }(i, j)}\left(t_{\text {route }}\right)= \\
& \quad\left(T_{\text {fire }}^{\text {cell }(i, j)}\left(t_{\text {route }}\right)-T_{m}^{\text {cell }(i, j)}\left(t_{\text {route }}\right)\right)
\end{aligned}
$$

\subsection{Decision for Optimal Intermediate Destination}

Based on utility maximization, the agent determines an optimal cell that could be an intermediate destination, with the consideration of minimizing the distance to the chosen exit (distance utility) based on the following rules:

- The intermediate destination has safe value greater than the precaution time, $T_{m}^{\text {prec }}$. The cells satisfying this rule are grouped in $A$ :

$$
A=\left\{\operatorname{cell}(i, j) \mid T \operatorname{safe} e_{m}^{\text {cell }(i, j)}>T_{m}^{\text {prec }}\right\}
$$


- The line $B_{m}^{(i, j)}$ connecting the agent $m$ with a chosen cell $(i, j)$ from $\mathrm{A}$, does not intersect cells of value less than the value of $T_{m}^{\text {prec }}$, The cells satisfying rule 1 and 2 are grouped in $C$ :

$$
C=\left\{\operatorname{cell}(i, j) \in A \mid B_{m}^{(i, j)} \cap\left\{\operatorname{cell}(i, j) \backslash \operatorname{Tsafe} e_{m}^{(i, j)} \leq T_{m}^{\text {prec }}\right\}=\varphi\right\}
$$

- The angle between the agent's direction toward the preferred destination and toward the intermediate destination is the minimum. The cells satisfying rule 1,2 , and 3 are grouped in $D$ :

$$
D=\left\{\operatorname{cell}(i, j) \in C \mid \min _{(i, j)}\left(\theta_{m \rightarrow \text { pref_destination }}^{m \rightarrow \text { cell(i, })}\right)\right\}
$$

- Finally, the optimal cell is determined as follow:

$$
\text { optimal_cell }=\text { index }\left\{\min _{(i, j) \in D} T s a f e_{m}^{(i, j)}\right\}
$$

The agent makes a decision to replace his preferred destination with the optimal cell as an intermediate destination:

$$
\vec{e}_{m}^{\text {pref }}(t)=\vec{e}_{m}^{\text {optimal_cell }}(t)
$$

\section{Simulation}

In this section, simulations are conducted in order to examine the evacuees' behavior regarding overtaking the fire and to test the efficiency of making smart and effective decisions during emergency evacuation scenarios. There are three steps in our simulation. The first time step is of size $\Delta t=.01 \mathrm{~s}$ used for the process of solving the system of differential equations of the SFM chosen based on enough accuracy for the model's reproduction. The second time step $\Delta t_{\text {route }}$ is used in the process of detecting intermediate points and making decision to select the optimal route. Last, the time step of size $\Delta t_{\text {exit }}$ is the assumed simple reaction, approximated to be equal to $2 \mathrm{~s}$ in [22]. Both $\Delta t_{\text {exit }}$ and $\Delta t_{\text {routes }}$ conform multiples of $\Delta t$. Because of the lack of relevant data, we use equal values for both $\Delta t_{\text {exit }}$ and $\Delta t_{\text {route }}$.

\subsection{Overtaking Behavior}

The first simulations in this section is to investigate an agents' behavior produced by the extended model against the fire. The situation of interest is the evacuation where two agents intend to leave a hall, as quickly as possible corresponding to their preferred velocities. The dimensions of the hall are 20 meters wide and 40 meters long. Margins along the walls are considered to provide agents space for reaching blocked exits by fire. Two exits are located on the low part of the right wall and right part of the upper wall. The location of the agents was initialized in the lower-left region of the hall, as shown in Figure 3. The fire is initialized in the lower-right part and prepared to spread according to simple probabilistic behavior (Figures 3, 4, and 5). The SSF is calculated according to Equation (12). The common values of the parameters used in the simulation were estimated as in Table 1.

Table 1. The description and values of the parameters used in the simulations.

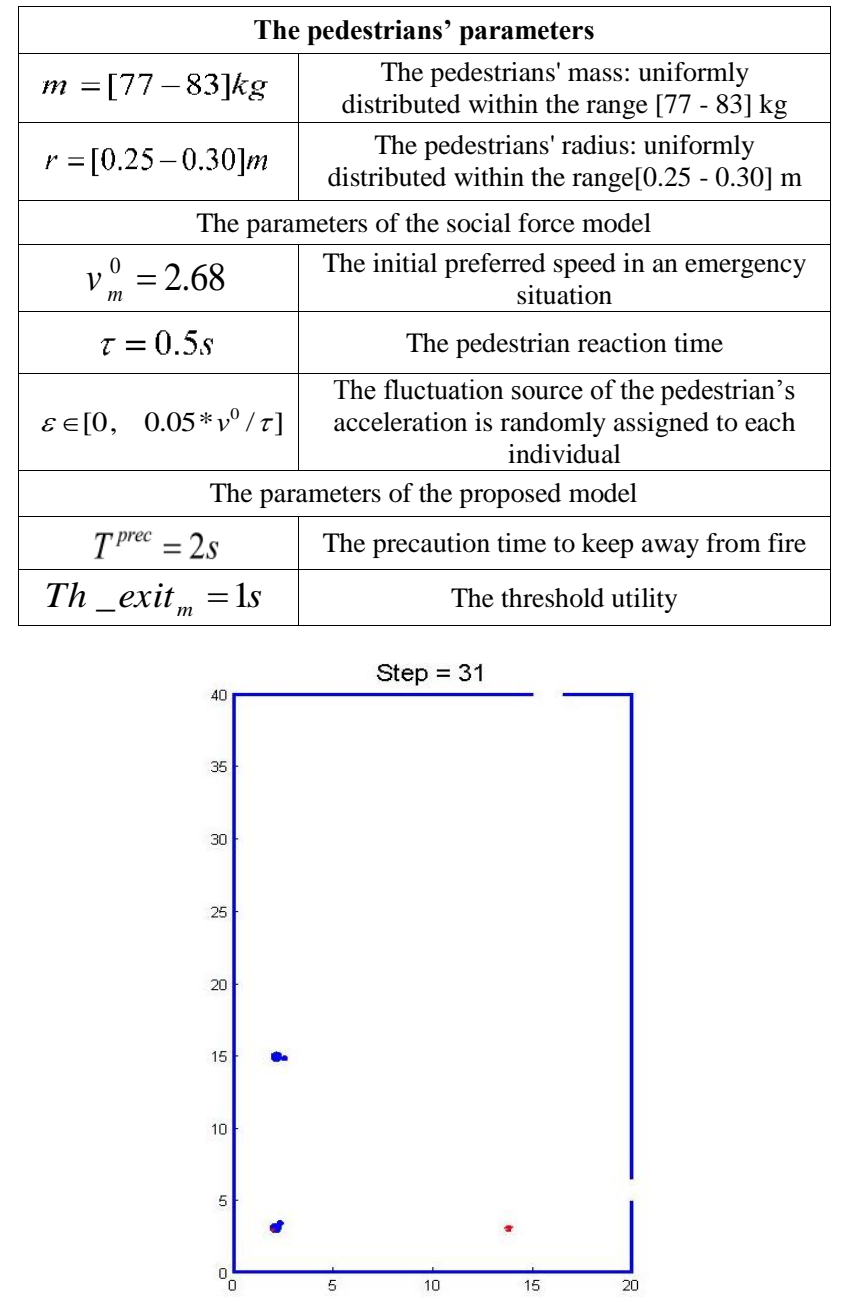

Figure 3. The initialization of two agents and their selection to the nearest exit (the lower exit).

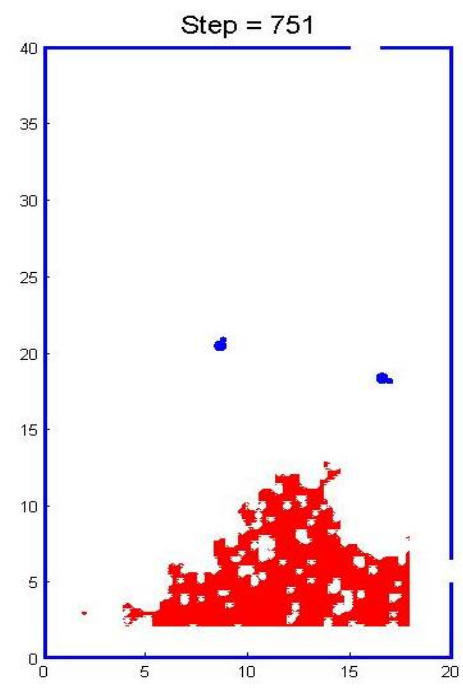

Figure 4 . The agent far away from the lower exit made a decision at the tactical level directing his movement towards the upper exit. 


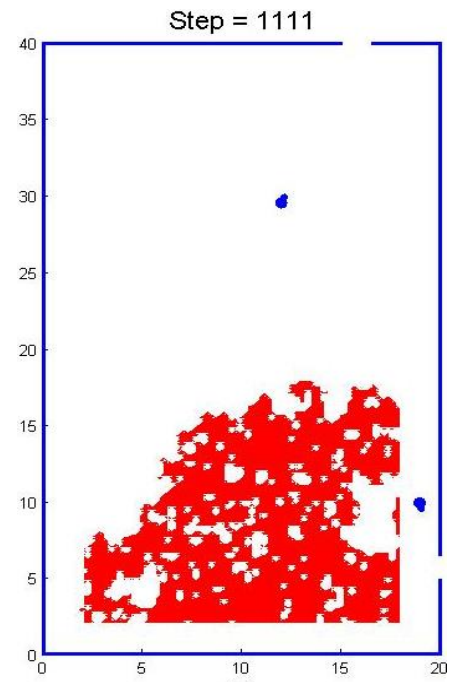

Figure 5. the first agent was able to overtake the fire and evacuate safely from his first chosen exit.

The results of the simulation reflect the agents' rational behavior when selecting their exits. In Figure 3, the agents selected the nearest exit (the lower exit). However, in Figure 4, the agent who was initialized near the lower wall was sticking to his choice because the overtaking process did not increase the disutility of the chosen exit greater than the other exit, whereas the second agent was exposed to critical location where the utility of exits became mostly equal. This situation led the agent to made a decision at the tactical level directing his movement towards the upper exit as shown in Figure 4. Finally, the first agent reached the margin which enabled him to evacuate safely as shown in Figure 5.

\subsection{Intelligent System for Efficient Evacuation}

The second simulations demonstrates the influence of incorporating intelligence capability into systems on obtaining typical evacuation. The setup of the simulations is similar to the above subsection, but the dimensions of the hall are 20 meters wide and 20 meters long. One hundred agents intend to leave the room as quickly as possible corresponding to their preferred velocity. We considered three different scenarios regarding the initialization of the agents' locations:

In the first scenario, the locations of the agents were initialized randomly in the entire hall as shown in Figure 6.

In comparison with the original exit choice model proposed in [22], we found similar initial behavior from the agents selecting exists with the highest utility. However, the extended model showed more intelligence in Figure 7, namely the agents who encounter the threatening fire are sticking with their choice as long as overtaking the fire does not cause greater disutility than the other exit. Such intelligent behavior keeps the initial optimal decisions made by the agents for long period of the evacuation time. Last, as shown in Figure 8, some agents were exposed to locations where the utility of exits became mostly equal, and therefore, decisions at the tactical level were made by those agents directing their motion toward the upper exit.

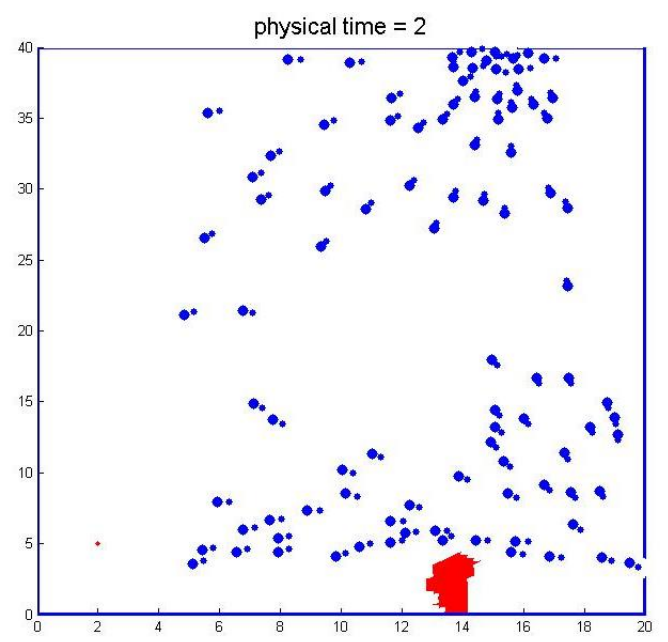

Figure 6. The initialization of 100 (distributed randomly in the entire hall) agents with the capability of overtaking the fire (the extended model).

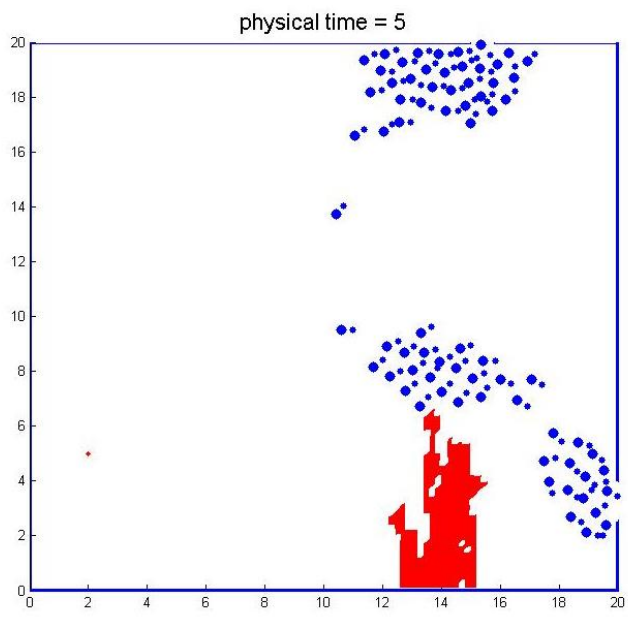

Figure 7. The agents in the extended model are sticking with their choice for longer period of time than the original model.

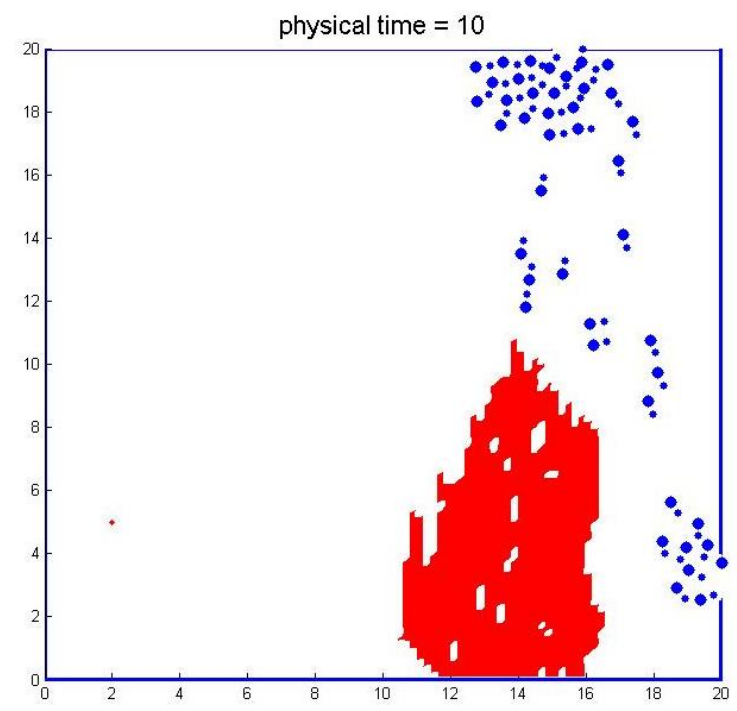

Figure 8. Decisions at the tactical level were made by those agents directing their motion toward the upper exit. 


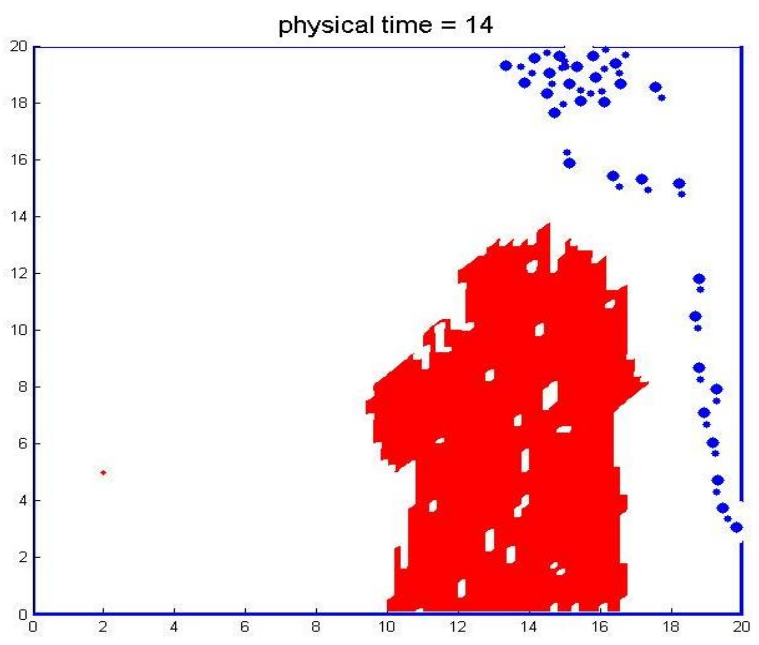

Figure 9. Efficient evacuation is performed by the extended model.

The decision process resulted in similar termination of the accumulated flows at the exits (see Figure 10-a). The original model, however, resulted in diverse termination of the accumulated flows at the exits as shown in Figure 10-b and less evacuated agents. Thus, the extended model performs more efficient evacuation. It is worth noting that the variant flows between the exits in both models, as illustrated in Figures 10-a and 10-b, refer to the higher accumulation at the upper exit than the right exit, so it caused the long-intermitted flow at the upper exit. The competition of the agents which is responsible for the faster-is-slower phenomenon is the main reason for this behavior $[8,27]$.

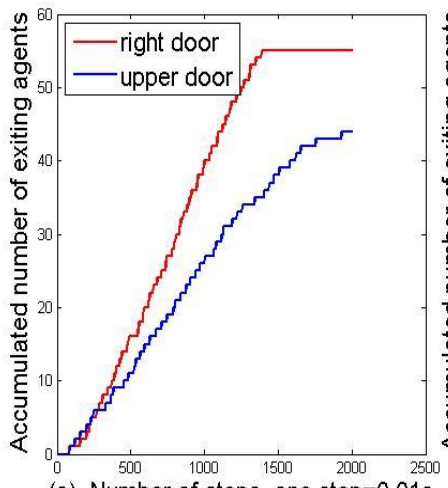

(a) Number of steps, one step $=0.01 \mathrm{~s}$ a) The extended model.

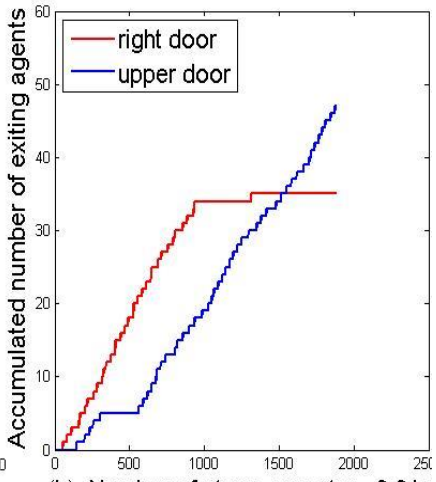

(b) Number of steps, one step $=0.01 \mathrm{~s}$ b) The original model.
Figure 10. The graphs show the accumulated number of the exiting individuals for all agents.

In the second scenario, we initialized the agents randomly in the lower region of the hall (biased toward the lower exit). Most agents initially selected the right exit because of its higher distance utility than the upper exit. However, compared with the first scenario (see Figures 7 and 8), faster decisions at the tactical level were made by greater number of agents directing their movement toward the upper exit (see Figure 11). The decision process resulted in further accumulation on the upper exit as shown in Figure 12, and therfore, some agents could not evacuate safely. However, compared with the original model in [22], the extended model still produce further efficiency because of the evacuation of some agents from the lower exit in the beginning of the simulation (see Figure 11).

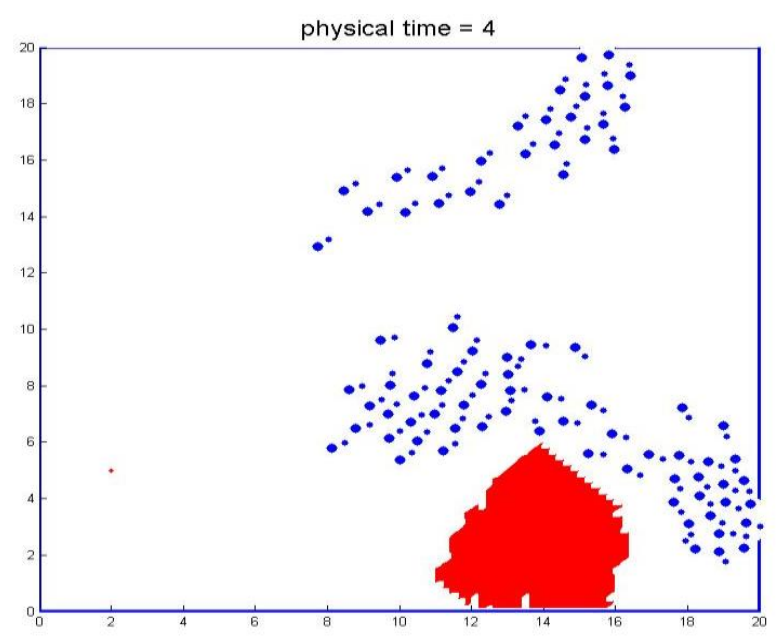

Figure 11. The evacuation of 100 agents distributed randomly in the lower region of the hall. Decisions at the tactical level were early made.

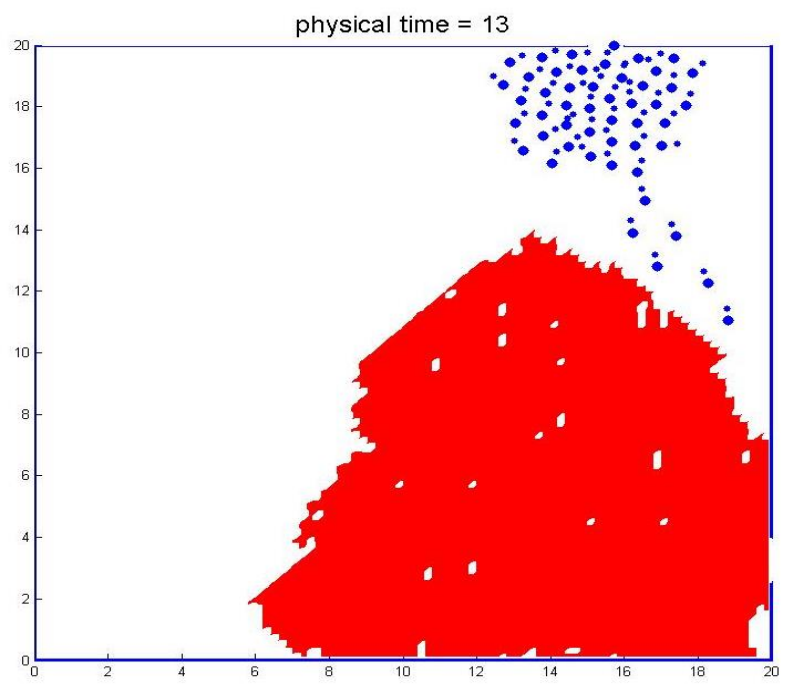

Figure 12. The end of the evacuation resulted in accumulation at the upper exit.

Last, we randomly initialized the agents in the upper region of the hall (biased toward the upper exit). In both models (original and extended), most agents initially selected the upper exit due to its higher distance-utility than the lower exit (see Figure 13). No overtaking behavior has been occurred because of the specification of this scenario.

The results from the considered three scenarios show that overtaking behavior enhances further the effectiveness of the evacuation when agents encounter the threatening source hindering their motion toward their chosen exit (the most efficient one). 


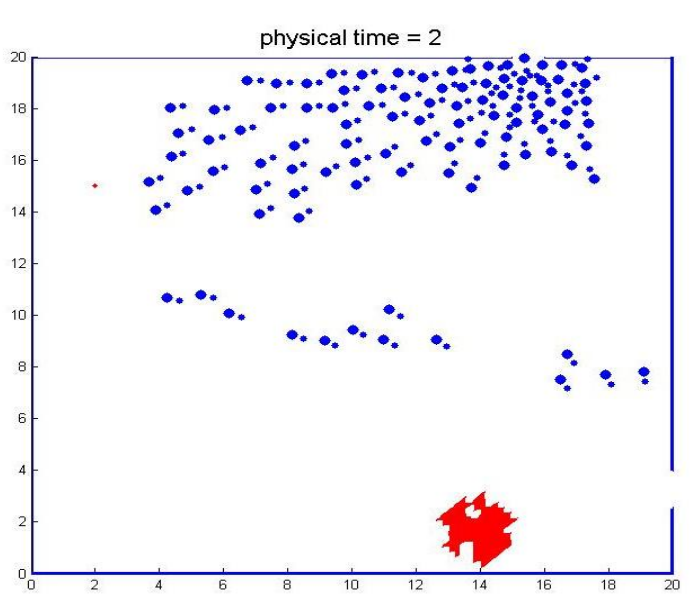

Figure 13. The evacuation of 100 agents distributed randomly in the upper region of the hall (the extended model).

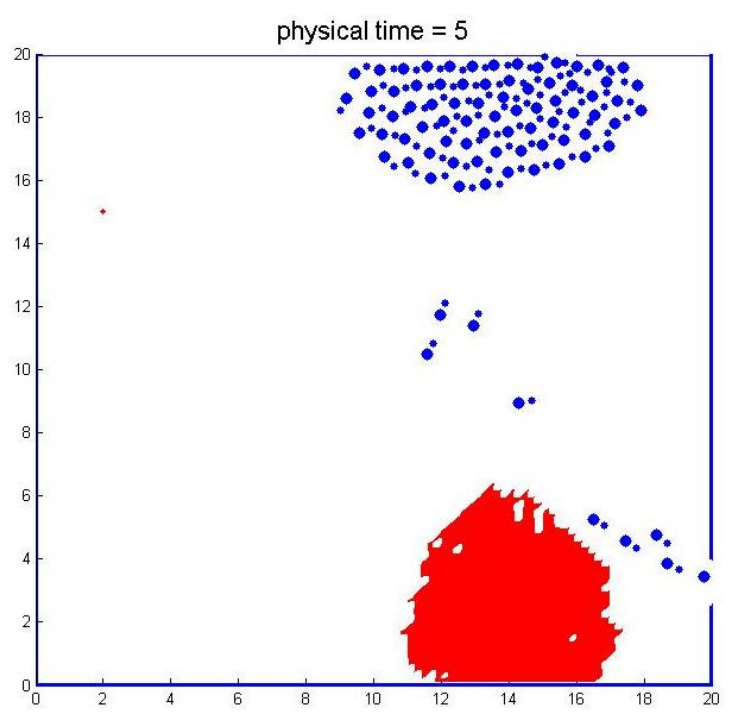

Figure 14. The end of the evacuation resulted in accumulation at the upped exit.

\section{Conclusions}

In this article, we modified an exit choice model based on time disutility by incorporating further intelligence capability. Namely, we endowed the simulated individuals with the ability to overtake the fire when the egress through the chosen exit is possible, and its utility is the highest. It is showed that the correct prediction of the threat caused by fire is crucial behavior which could lead to survival, and that right decision timing of giving up selecting a chosen exit for avoiding fire risk is a crucial factor. Although the delayed decision could threaten the safety of an individual, earlier decisions could result in disorder and further blockage of the alternative exit, and consequently, lengthen the evacuation time.

The results indicate the importance of developing a decision-support system for crowd management to provide the evacuees with intelligence using different instruments be they computational (signals) or noncomputational instruments (informed security to work as leaders).

\section{Acknowledgment}

This research was supported by the Deanship of Scientific Research, Imam Mohammad Ibn Saud Islamic University, Saudi Arabia, Grant No. (19-12-08001).

\section{References}

[1] Blue V. and Adler J., "Cellular Automata MicroSimulation of Bi-Directional Pedestrian Flows," Journal of the Transportation Research Board, vol. 1678, no.1, pp. 135-141, 1999.

[2] Bohannon J., "Directing the Herd: Crowds and the Science of Evacuation," Science, vol. 310, no. 5746, pp. 219-221, 2005.

[3] Burstedde C., Klauck K., Schadschneider A., and Zittartz J., "Simulation of Pedestrian Dynamics Using a Two-Dimensional Cellular Automaton," Physica A: Statistical Mechanics and its Applications, vol. 295, no. 3-4, pp. 507-525, 2001.

[4] Cao S., Song W., Liu X., and Mu N., "Simulation of Pedestrian Evacuation in A Room under Fire Emergency," Procedia Engineering, vol. 71, pp. 403-409, 2014.

[5] Cristiani E., Piccoli B., and Tosin A., Multiscale Modeling of Pedestrian Dynamics, Springer, 2014.

[6] Guo N., Jiang R., Hu M., and Ding J., "Constant Evacuation Time Gap: Experimental Study and Modeling," Chinese Physics B, vol. 26, no. 12, 2017.

[7] Gwynne S., Hulse L., and Kinsey M., "Guidance for the Model Developer on Representing Human Behavior in Egress Models," Fire Technology, vol. 52, no. 3, pp. 775-800, 2016.

[8] Helbing D., Farkas I., and Vicsek T., "Simulating Dynamical Features of Escape Panic," Nature, vol. 407, no. 6803, pp. 487-490, 2000.

[9] Helbing D., Farkas I., Molnár P., and Vicsek T., "Simulation of Pedestrian Crowds in Normal and Evacuation Situations," Pedestrian and Evacuation Dynamics, vol. 21, no. 2, pp. 21-58, 2002.

[10] Helbing D. and Molnár P., "Social Force Model for Pedestrian Dynamics," Physical Review E, vol. 51, no. 5, pp. 4282-4286, 1995.

[11] Huang H. and Guo R., "Static Floor Field and Exit Choice for Pedestrian Evacuation in Rooms with Internal Obstacles and Multiple Exits," Physical Review E, vol. 78, no. 2, 2008.

[12] Hughes R., "Continuum Theory for the Flow of Pedestrians," Transportation Research Part B: Methodological, vol. 36, no. 6, pp. 507-535, 2002.

[13] Jaber K., Alia O., and Shuaib M., "P-HS-SFM: A Parallel Harmony Search Algorithm for the Reproduction of Experimental Data in the Continuous Microscopic Crowd Dynamic 
Models," Journal of Experimental and Theoretical Artificial Intelligence, vol. 30, no. 2, pp. 235-255, 2018.

[14] Keating J., "The Myth of Panic," Fire Journal, vol. 76, no. 3, pp. 57-61, 1982.

[15] Kinateder M., Comunale B., and Warren W.H., "Exit Choice in an Emergency Evacuation Scenario Is Influenced By Exit Familiarity and Neighbor Behavior," Safety Science, vol. 106, pp. 170-175, 2018.

[16] Kirchner A. and Schadschneider A., "Simulation of Evacuation Processes Using A Bionics-Inspired Cellular Automaton Model For Pedestrian Dynamics," Physica A: Statistical Mechanics And Its Applications, vol. 312, no. 1-2, pp. 260-276, 2002.

[17] Kuligowski E., Peacock R., and Hoskins B., A Review of Building Evacuation Models Technical Note (NIST TN), Gaithersburg, 2010.

[18] Lakoba T.I., Kaup D.J., and Finkelstein, N.M., "Modifications of the Helbing-Molnar-FarkasVicsek Social Force Model For Pedestrian Evolution," Simulation, vol. 81, no. 5, pp. 339352, 2005.

[19] Luh P., Wilkie C., Chang S., Marsh K., and Olderman N., "Modeling and Optimization of Building Emergency Evacuation Considering Blocking Effects on Crowd Movement," IEEE Transactions on Automation Science and Engineering, vol. 9, no. 4, pp. 687-700, 2012.

[20] Proulx, G., "A Stress Model for People Facing a Fire," Journal of Environmental Psychology, vol. 13, no. 2, pp. 137-147, 1993.

[21] Ronchi E. and Nilsson D., "Basic Concepts and Modelling Methods," Evacuation Modeling Trends, Springer, 2016.

[22] Shuaib M., "Incorporating Intelligence for Typical Evacuation under the Threat of Fire," Safety Science, vol. 106, pp.1-9, 2018.

[23] Shuaib M., "Preserving Socially Expected Crowd Density in front of the Exit for the Reproduction of Experimental Data by Modeling the Pedestrians' behind Perception," Journal of Statistical Mechanics: Theory and Experiment, vol. 2014, no. 10, 2014.

[24] Shuaib M., "Modeling the Capability of Penetrating Jammed Crowd to Eliminate Freezing Transition," Chinese Physics B, vol. 25, no. 5, 2016.

[25] Shuaib M., "Modeling Collision Avoidance Field for Overtaking Moving Obstacles," in Proceedings of the International Arab Conference on Information Technology, Al Ain, pp. 102-106, 2019.

[26] Sime J., "Crowd Facilities, Management and Communications in Disasters," Facilities, vol. 17, no. 9/10, pp. 313-324, 1999.
[27] Zhang G., Huang D., Zhu G., and Yuan G., "Probabilistic Model for Safe Evacuation under the Effect of Uncertain Factors in Fire," Safety Science, vol. 93, pp. 222-229, 2017.

[28] Zheng Y., Jia B., Li X.G., and Zhu N., "Evacuation Dynamics with Fire Spreading Based on Cellular Automaton," Physica A: Statistical Mechanics and its Applications, vol. 390, no. 1819, pp. 3147-3156, 2011.

[29] Zheng Y., Jia B., Li X., and Jiang R., "Evacuation Dynamics Considering Pedestrians' Movement Behavior Change with Fire and Smoke Spreading," Safety Science, vol. 92, pp. 180-189, 2017.

[30] Zhu J., Li W., Li H., Wu Q., and Zhang L., “A Novel Swarm Intelligence Algorithm for the Evacuation Routing Optimization Problem," The International Arab Journal of Information Technology, vol. 14, no. 6, pp. 880-889, 2017.

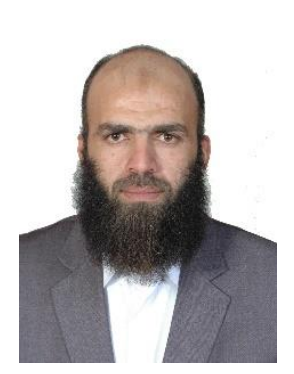

Mohammed Shuaib received Ph.D. Degree in Applied Mathematics, Universiti Sains Malaysia, in 2011. Field: Applied Mathematics; Mathematical Modeling; M. Sc. Degree in Mathematics, University of Jordan, in 2003; B.A. Degree in Educational sciences/ field teacher (Mathematics), University of Jordan, in 1999. From 2011 to 2018, he had been an Assistant Professor of mathematics at the Department of Computer Sciences, College of Shari'a and Islamic Studies in Al Ahsaa, Imam Mohammad Ibn Saud Islamic University (IMSIU). Currently, he is working as an Associate Professor in the same university. His current interests include modeling and simulation of crowd dynamics. His current research is about developing a crowd simulation to present the real aspects of the Hajj Crowd.

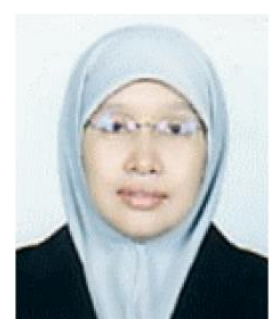

Zarita Zainuddin received the B. S. degree in Mathematics from Monmouth College, USA in 1979, M. Sc. in Applied Mathematics from Ohio University, USA in 1981, M. Sc. in Mathematics (Control Theory) from UMIST, UK in 1986 and Ph.D from Universiti Sains Malaysia in 2001. She is currently a Professor at the School of Mathematical Sciences, Universiti Sains Malaysia, Penang, Malaysia. Her current interests include neural networks, image processing, bioinformatics and crowd dynamics. She focuses on the improvement and development of neural network learning algorithms involving incorporation of acceleration and optimization methods into the training of neural networks for improved accuracy and convergence. 